\title{
Coulomb explosion imaging for the visualization of a conical intersection
}

M. E. Corrales ${ }^{1,2}$, J. González-Vázquez ${ }^{3}$, R. de Nalda $^{4 *}$, L. Bañares ${ }^{1}$

${ }^{1}$ Departamento de Química Física (Unidad Asociada I+D+i al CSIC), Facultad de Ciencias Químicas, Universidad Complutense de Madrid, 28040 Madrid, Spain

${ }^{2}$ Centro de Láseres Ultrarrápidos, Facultad de Ciencias Químicas, Universidad Complutense de Madrid, 28040 Madrid, Spain

${ }^{3}$ Departamento de Química and Institute for Advanced Research in Chemical Sciences (IAdChem), Módulo 13, Facultad de Ciencias, Universidad Autónoma de Madrid, 28049 Madrid, Spain

${ }^{4}$ Instituto de Química Física Rocasolano, CSIC, C/ Serrano 119, 28006 Madrid, Spain

*Corresponding author: r.nalda@csic.es 


\section{ABSTRACT}

Coulomb explosion imaging is proposed as a method to directly map the presence of conical intersections encountered by a propagating wave packet in a molecular system. The case of choice is the non-adiabatic coupling between two dissociative surfaces in the methyl iodide molecule, probed by Coulomb explosion with short, intense near-infrared pulses causing multiple ionization. On-the-fly multidimensional trajectory calculations with surface hopping using perturbation theory and including spin-orbit coupling are performed to visualize the dynamics through the conical intersection and compare with experimental results. The possibilities and limitations of the technique are examined and discussed.

TOC GRAPHICS

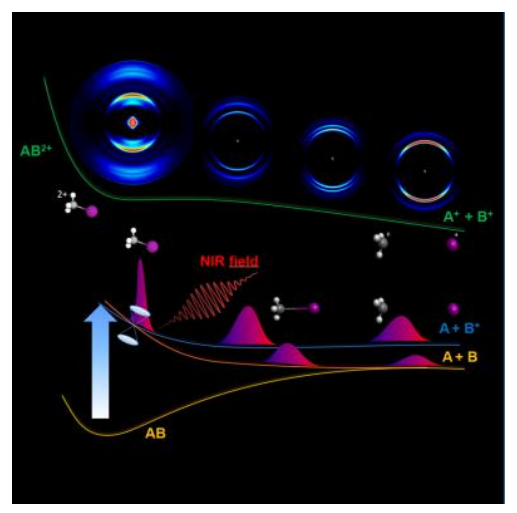

KEYWORDS: Nonadiabatic processes, ultrafast dynamics, methyl halides, strong laser fields, surface hopping. 
A central ingredient of most chemists' and physicists' intuitive understanding of molecular dynamics is based on the idea that atomic nuclei move in one potential energy hypersurface created by the much faster electron cloud, this idea being at the core of the BornOppenheimer approximation that is so powerful to describe near-equilibrium or in general adiabatic processes. However, it is well known that the dynamics on excited states presents a higher complexity and is often governed by conical intersections (CI) intersections ${ }^{1-3}$ or intersystem crossings. As a result, non-adiabatic transitions ${ }^{4-5}$ play a central role on a plethora of physicochemical processes, like radiationless decay or charge transfer.

When a molecular wave packet explores a CI during its propagation, a superposition is created where the wave packet propagates coherently in both surfaces involved. The signatures of the influence of conical intersections in experimental data tend to be of spectroscopic nature, i.e. indirect in the sense that considerable previous knowledge is required on the potential energy surfaces and the excitation mechanism, so that observables can be correlated with the presence of the conical intersection. In this way, time-resolved spectroscopy can reveal conical intersections through the measurement of changes in absorption spectra ${ }^{6}$, photoelectron spectra ${ }^{7}$ or polarization anisotropy ${ }^{8}$.

In this work, we use Coulomb explosion ${ }^{9-13}$ for the observation of the presence of a conical intersection in a manner that we will call "direct" in the sense that it is both time-resolved and through the detection of an observable that is not spectroscopic but a direct mapping of the coordinate space. Coulomb explosion occurs when several electrons are ejected from a molecular moiety, and its charged components undergo explosion due to electrostatic repulsion. The complete measurement of the final velocity vectors of the resulting fragments can provide information on the geometry of molecules ${ }^{14}$ or clusters ${ }^{15-16}$, the 
alignment or orientation of a molecular ensemble ${ }^{17-18}$, and even provides a tool for the identification of isomers ${ }^{19}$ or enantiomers ${ }^{20}$. Since Coulomb explosion can be conceived as a notably direct method to measure internuclear distances through the measurement of final fragment kinetic energies, its application to the real-time study of dynamic molecular processes like vibration, dissociation or isomerization is straightforward provided that a source of light capable of removing several electrons from the moiety in a sufficiently short time is available. Since the first demonstration of femtosecond time-resolved laser-induced Coulomb explosion used to diagnose the wave packet dynamics in a photodissociation event $^{21}$, the technique has been quickly acquiring maturity over recent years ${ }^{9-12,22-24}$.

One of the prototype conical intersections in photodynamics is that in the $\mathrm{CH}_{3} \mathrm{I}$ molecule, occurring between two of the surfaces of the manifold of strongly dissociative spin-orbit states for which an electron in a nonbonding $p$ orbital in the iodine atom is promoted to the lowest energy available antibonding orbital on the C-I bond. UV excitation from the molecular ground state, spectroscopically called the $A$-band, leads to a transition solely to one of the surfaces in this manifold, termed ${ }^{3} Q_{0}$ in Mulliken notation (see Figure 1). Excitation of the ${ }^{3} Q_{0}$ surface adiabatically leads to the formation of the iodine atom in its spin-orbit excited state, $\mathrm{I}^{*}\left({ }^{2} P_{1 / 2}\right)$. Since ground state iodine $\mathrm{I}\left({ }^{2} P_{3 / 2}\right)$ is also found as a product of this transition, a non-adiabatic coupling between the ${ }^{3} Q_{0}$ surface and the ${ }^{1} Q_{1}$ surface -leading to $\mathrm{I}\left({ }^{2} P_{3 / 2}\right)$ - must exist. This has been described in detail in the past, both in asymptotic $^{25-27}$ and time-resolved studies ${ }^{28-30}$. Several theoretical studies have been performed in this molecule to evaluate the effect of the conical intersection in the photodissociation dynamics. Most of the previous calculations were based on potential 
energy surfaces parameterized by Amatatsu et al. ${ }^{31-32}$ and later corrected by Xie et al. ${ }^{33}$. Beyond the original surface hopping dynamics, several quantum dynamical simulations were performed including three ${ }^{29,33-34}$, four ${ }^{30}$ or even the nine ${ }^{35}$ degrees of freedom of the molecule.

In this work, we propose Coulomb explosion imaging (CEI) as a method to directly map the presence of conical intersections explored by an evolving wave packet. Kinetic energies of the exploding fragments carry the $1 / r$ dependence of the Coulomb energy as a function of the internuclear distance, and this is used to map their dynamics in the coordinate space through time-resolved detection. In the experiment described here, real-time probing of the $A$-band dissociation of $\mathrm{CH}_{3} \mathrm{I}$ with an intense NIR laser pulse causes the ejection of more than one electron in the dissociating moiety, which leads to Coulomb explosion due to repulsion between its positively charged components. Since the first stages of $A$-band dissociation witness a conical intersection between two excited surfaces (see Figure 1), measurement of the resulting charged fragments has the capability to map the wave packet branching in this region. 


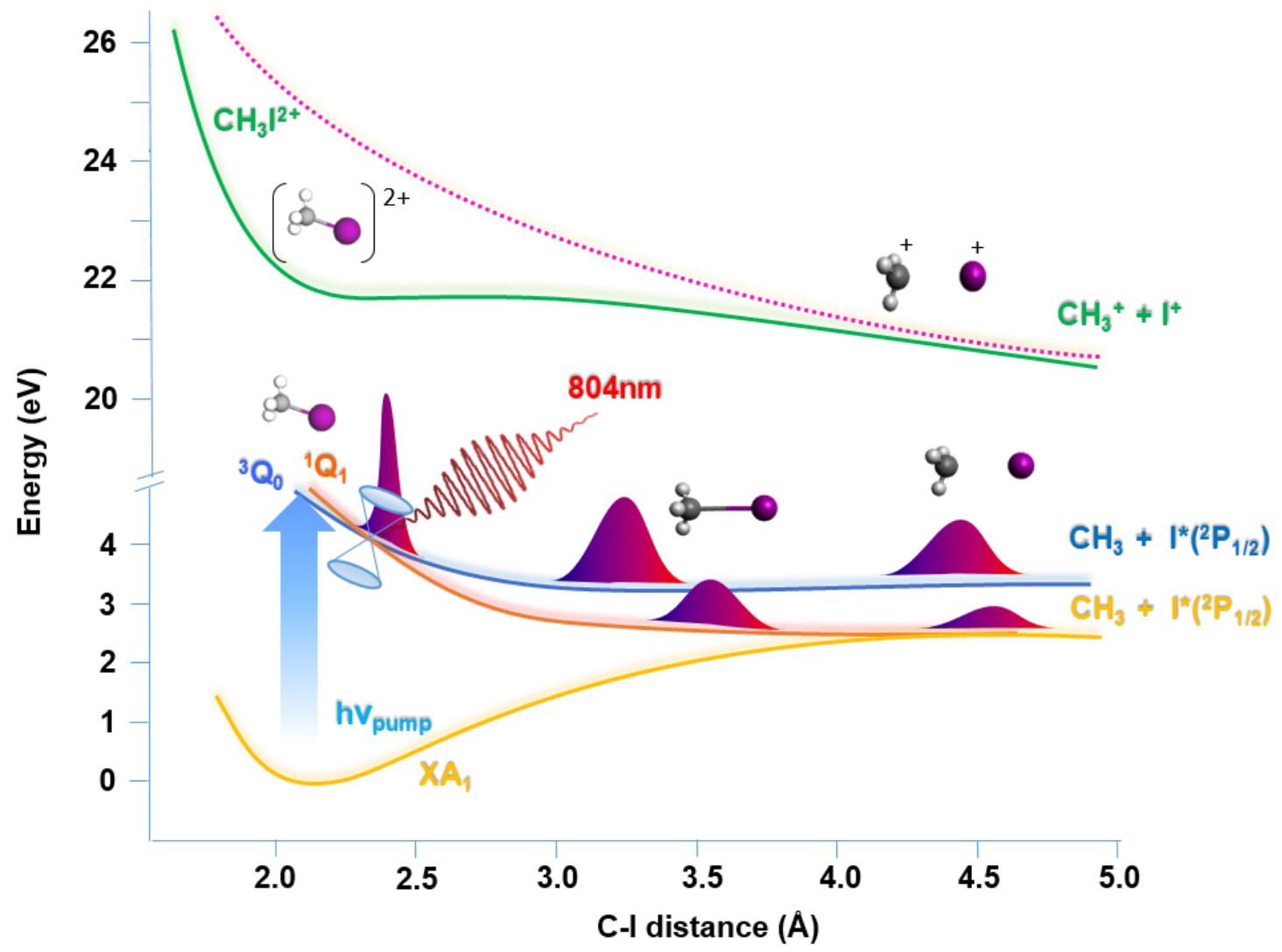

Figure 1. Diagram of the potential energy surfaces involved in the Coulomb explosion probing of $A$-band dissociation in $\mathrm{CH}_{3} \mathrm{I}$. The excited neutral surfaces present a crossing, indicated with a double cone, where the wave packet, initially created in the ${ }^{3} Q_{0}$ potential energy surface (in blue) branches to yield iodine atoms in different spin orbit states. The wave packet is projected into the dication states by extraction of two electrons with an intense NIR field. The dication ground state is plotted in green. The purely Coulombic surface between two point charges is plotted in a pink dashed line for reference.

The strong NIR field alone, in the absence of the UV field, causes both dissociative ionization (DI) and Coulomb explosion (CE) of the $\mathrm{CH}_{3} \mathrm{I}$ molecule. The signatures of these 
two processes can be distinguished in the top left panel of Figure 2, where Abel-inverted $\mathrm{CH}_{3}{ }^{+}$images are shown for these conditions. The central, low kinetic energy region corresponds to the DI process, whereas the high kinetic energy rings are CE channels that were already documented ${ }^{36-37}$, and were attributed to excitation to different electronic states of the $\mathrm{CH}_{3} \mathrm{I}^{2+}$ dication ${ }^{36}$. All the other panels in Figure 2 correspond to Abel-inverted $\mathrm{CH}_{3}{ }^{+}$ images obtained upon irradiation with UV and $2 \times 10^{13} \mathrm{~W} / \mathrm{cm}^{2} \mathrm{NIR}$ pulses at specific time delays. For those, subtraction of the only-NIR image was applied for better distinction. Images directly obtained without subtraction are included in the Supporting Information (Figure S1). The features that appear in this sequence of images are the following: (a) For all images after time zero, a clear ring of parallel anisotropy appears, marked as "DISS" (for "dissociation") on the Figure. This ring is accompanied by a weaker ring of larger radius. These two rings correspond to the UV $A$-band photodissociation of $\mathrm{CH}_{3} \mathrm{I}$ with its two well known channels leading to $\mathrm{CH}_{3}+\mathrm{I} *\left({ }^{2} P_{1 / 2}\right) / \mathrm{I}\left({ }^{2} P_{3 / 2}\right)$, followed by non-resonant ionization of the resulting $\mathrm{CH}_{3}$ fragment by the NIR pulse. In the second panel, acquired near time zero, this contribution appears enhanced and with higher anisotropy. This behavior was described in Refs. ${ }^{38-40}$. (b) There is a feature that only appears in the second panel, at delay time $50 \mathrm{fs}$, and it is marked as "LICI" (for "light-induced conical intersection"). This was studied in detail in Ref. ${ }^{40}$ and was attributed to the appearance of a new channel through the creation of a LICI in $\mathrm{CH}_{3} \mathrm{I}$.

(c) The main characteristic of using higher intensity NIR fields are the two additional rings, one notably more intense than the other, with diminishing radius as time grows, which appear in all images. They are marked as "DCE" (for "Dynamic Coulomb Explosion") in 
the Figure. These are Coulomb explosion rings in the dissociating $\mathrm{CH}_{3} \mathrm{I}$ molecule and constitute the main interest of the present work.

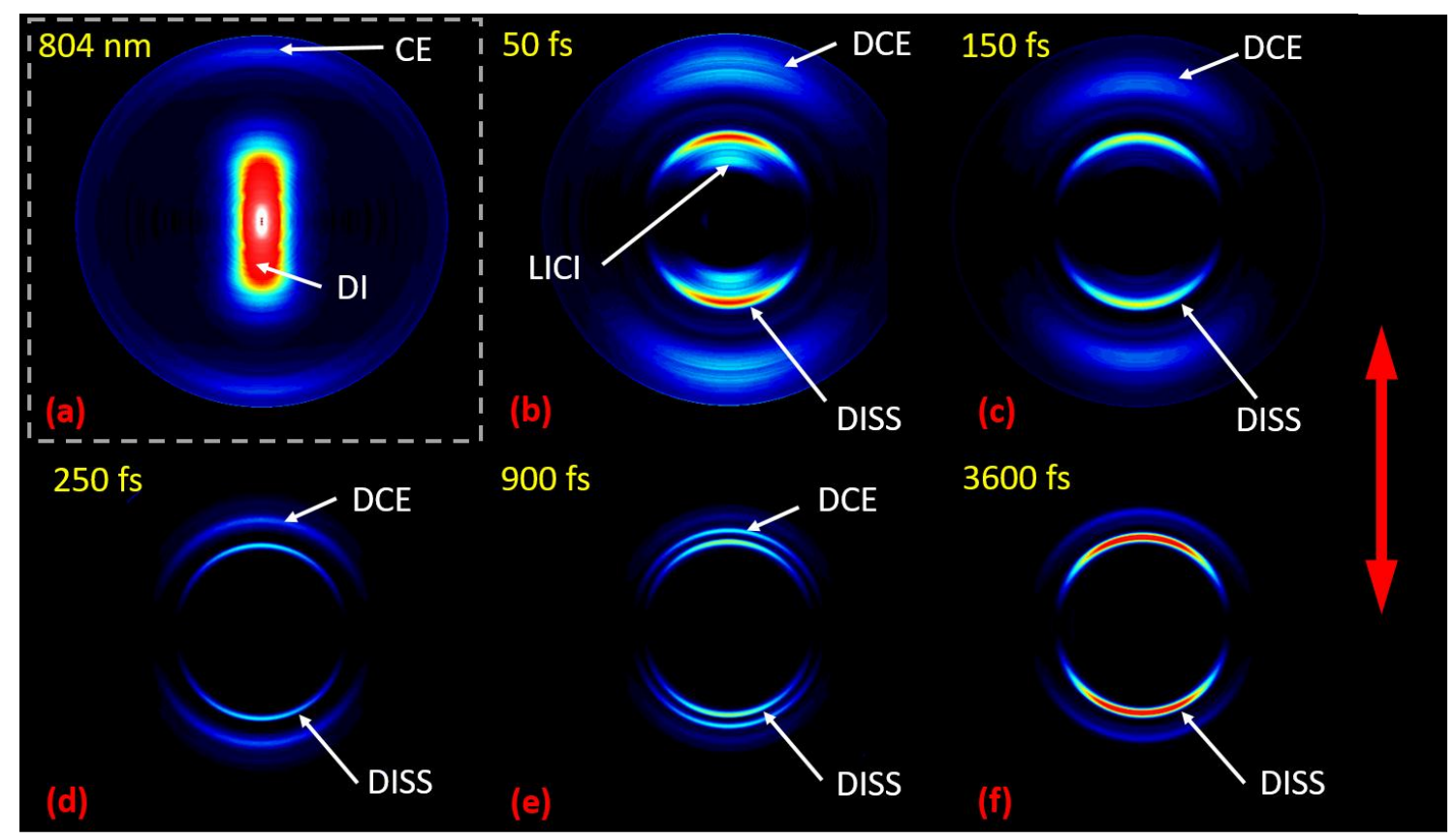

Figure 2. (a) Abel-inverted $\mathrm{CH}_{3}{ }^{+}$image obtained with only the NIR pulse present. (b)-

(f) Sequence of Abel-inverted $\mathrm{CH}_{3}{ }^{+}$images obtained at selected pump-probe time

delays after subtraction of the images obtained with the NIR pulses alone. The double arrow indicates the polarization direction of pump and probe beams. Labels for the observed channels are CE: Coulomb explosion; DI: dissociative ionization; DISS: dissociation; LICI: light-induced conical intersection; DCE: dynamic Coulomb explosion. See the text for details.

Angular integration of the Abel-inverted $\mathrm{CH}_{3}{ }^{+}$images yields the total kinetic energy release (KER) distributions for each time delay. These can be plotted as a 2D map as a function of 
time, as is shown in Figure 3. The contributions identified in the images are indicated with the same labels on this map. The map corresponds to performing NIR-only subtraction for each case (the original map is included in Figure S2 of the Supporting Information). The solid lines represent calculated central kinetic energies expected for the DCE channels considering a classical 1D model, where the neutral surfaces ${ }^{3} Q_{0}$ (white curve) and ${ }^{1} Q_{1}$ (blue curve) are obtained from Ref. ${ }^{32}$. Since UV absorption at $268 \mathrm{~nm}$ is assumed to occur exclusively to the ${ }^{3} Q_{0}$ surface, the white line is drawn from time zero and the blue line is only drawn from the time at which the classical trajectory (see below) finds the crossing between the ${ }^{3} Q_{0}$ and ${ }^{1} Q_{1}$ surfaces. Figs. $3 \mathrm{~b}$ and $3 \mathrm{c}$ show a zoomed version of the short time region, and the difference between the panels lies on the method for the calculation of the solid lines. In Fig. 3c, they are calculated assuming a purely coulombic potential for two point charges located in the center of mass of the $\mathrm{CH}_{3}{ }^{+}$and the $\mathrm{I}^{+}$cations. As can be seen, a good agreement is obtained for most of the trajectory, but a clear discrepancy is visible for very short time delays, where this method overestimates the resulting kinetic energies. Contrarily, in Fig. 3b, and in Fig. 3a, the calculated 1D potential for the dication ground state $^{36}$ was used to calculate kinetic energies. It had already been found ${ }^{36}$ that an important stabilization occurs for the dication at short internuclear distances and a purely coulombic curve does not accurately represent the behavior in this region. The fact that, somewhat counterintuitively, the calculated kinetic energy initially increases with time, is related to the fact that the 1D slope of the excited neutral surfaces at the Franck-Condon region is locally higher than that of the $\mathrm{CH}_{3} \mathrm{I}^{2+}$ dication, and thus the gain in kinetic energy upon neutral dissociation is initially higher than that from Coulomb explosion. It is clear that the agreement with the data when the calculated dication surface is taken into account is significantly better. Equivalent data obtained for the $\mathrm{I}^{+}$fragment are shown in Figures S3, 
S4 and S5 in the Supporting Information. The channels observed through the observation of I+ are the same but energy resolution is worse, since the voltages applied in the velocity map imaging apparatus were kept identical.

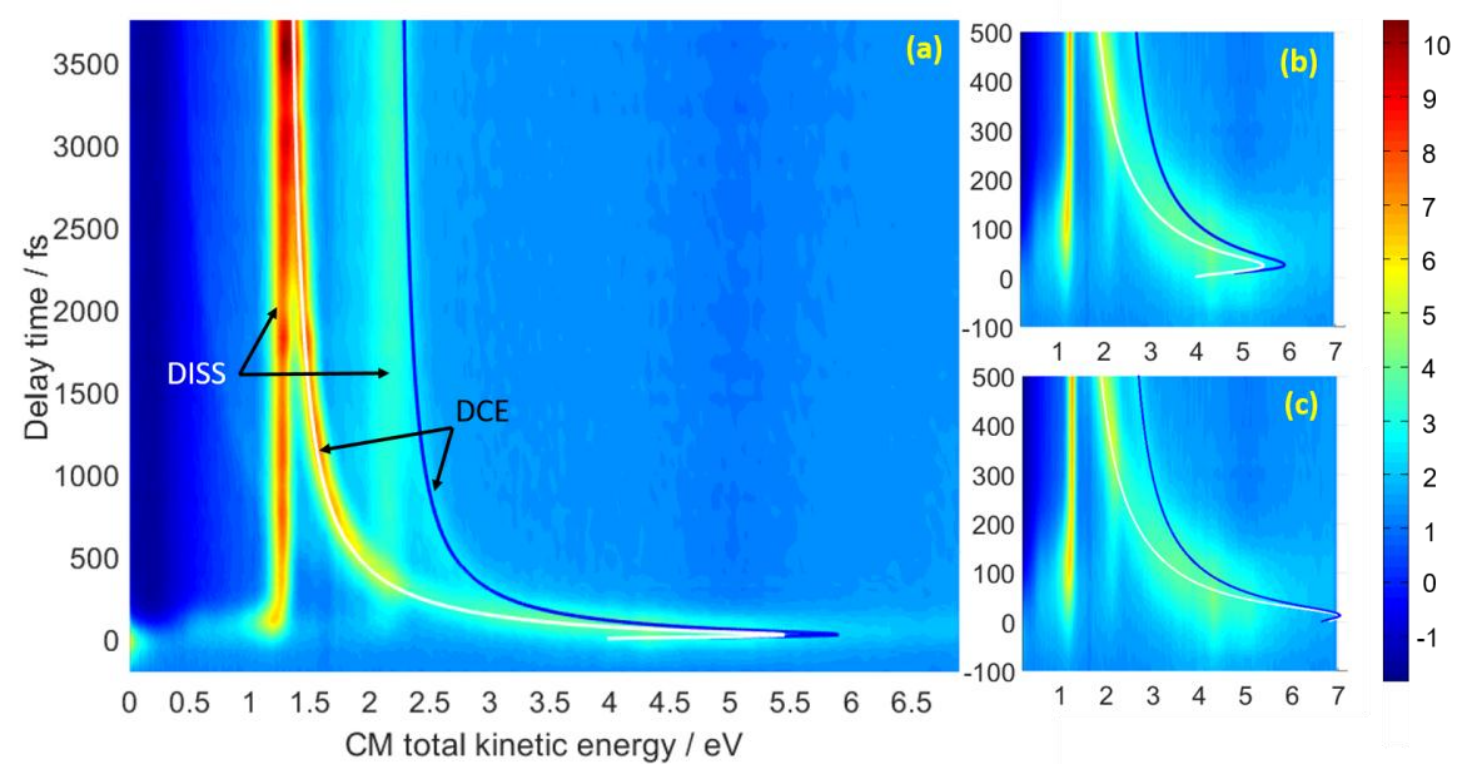

Figure 3. (a) 2D false-color map containing the set of center-of-mass (CM) total KER, as a function of time delay, obtained from $2 \pi$ angular integration of the series of Abelinverted $\mathrm{CH}_{3}{ }^{+}$images like those shown in Figure 2. The contribution obtained upon irradiation with NIR-only pulses has been subtracted. The channels identified and described in the text are marked on the map. (b)-(c) Zoom of the short delay region of (a). In all cases, the solid lines indicate calculated kinetic energies obtained with a 1D classical model (see the text for details).

The results of the ab initio on-the-fly trajectory calculations performed for this system are shown in Figure 4, where color changes (from red to blue) indicate surface hopping events. 
Fig. 4a shows the evolution of the carbon-iodine distance as a function of time after excitation to the excited neutral surfaces. Fig. $4 \mathrm{~b}$ shows the behavior of the bending angle I$\mathrm{C}-\mathrm{H}_{3}$, that breaks the $\mathrm{C}_{3 \mathrm{v}}$ symmetry, as a function of the internuclear distance during dissociation. As can be seen, this angle, initially confined to a narrow region around $105^{\circ}$, evolves to a much broader distribution when the two fragments separate.

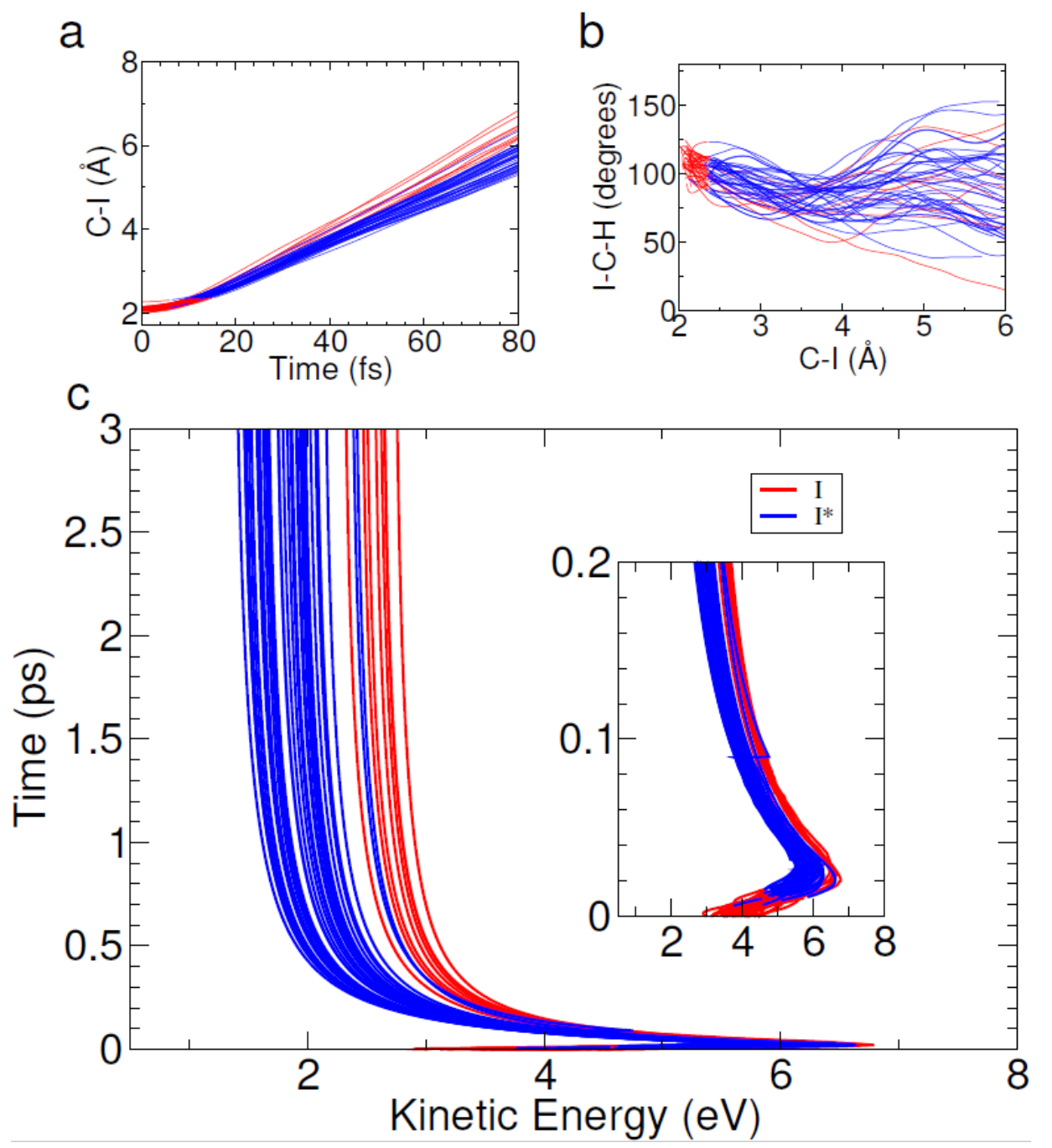

Figure 4. Trajectory surface-hopping (red the lowest 8 potentials correlating with I and blue the highest 4 potentials correlating with $I^{*}$ ) adiabatic description of the 
dissociation dynamics. See the theoretical section for more details. (a) Time evolution of the carbon-iodide distance. (b) Time evolution of the bending angle that breaks the $\mathrm{C}_{3 \mathrm{v}}$ symmetry. (c) Simulation of the Coulomb explosion energy during the dynamics.

Figure $4 \mathrm{c}$ contains a simulation of the Coulomb explosion energy during the dynamics from the time of excitation to 3 picoseconds for each of the computed trajectories. Quasicoulombic behavior is shown for most of the trajectory. The initial (counterintuitive) growth of kinetic energy with time, already reported for the 1D simulations plotted in Figure 3, is confirmed for the more sophisticated simulation applied here. A zoomed plot with the first $200 \mathrm{fs}$ of evolution in shown in the inset of Fig. 4c, where it is clear that all trajectories experience this phenomenon.

The inset of Fig. 4c shows the region where the trajectories are affected by surface hopping, i.e. the region of the non-adiabatic crossing. In the semiclassical trajectories, crossing takes place approximately 10-20 fs after excitation. We have calculated a semiclassical trajectory for the two channels of the photodissociation reaction by averaging the 50 trajectories, and a direct comparison between this averaged trajectory and the experimental results is depicted in Figure 5. As can be seen, the agreement is very good. The theoretical simulation reveals the position of the conical intersection at $(4.9 \mathrm{eV}, 13 \mathrm{fs})$. Experimentally, this is a region somewhat blurred by the cross correlation of the excitation and Coulomb explosion lasers; moreover, the very rapid coordinate-dependent changes of the potential energy surfaces in this region make discrimination even more challenging. However, the bifurcation of the Coulomb trajectories clearly identifies the conical intersection. 


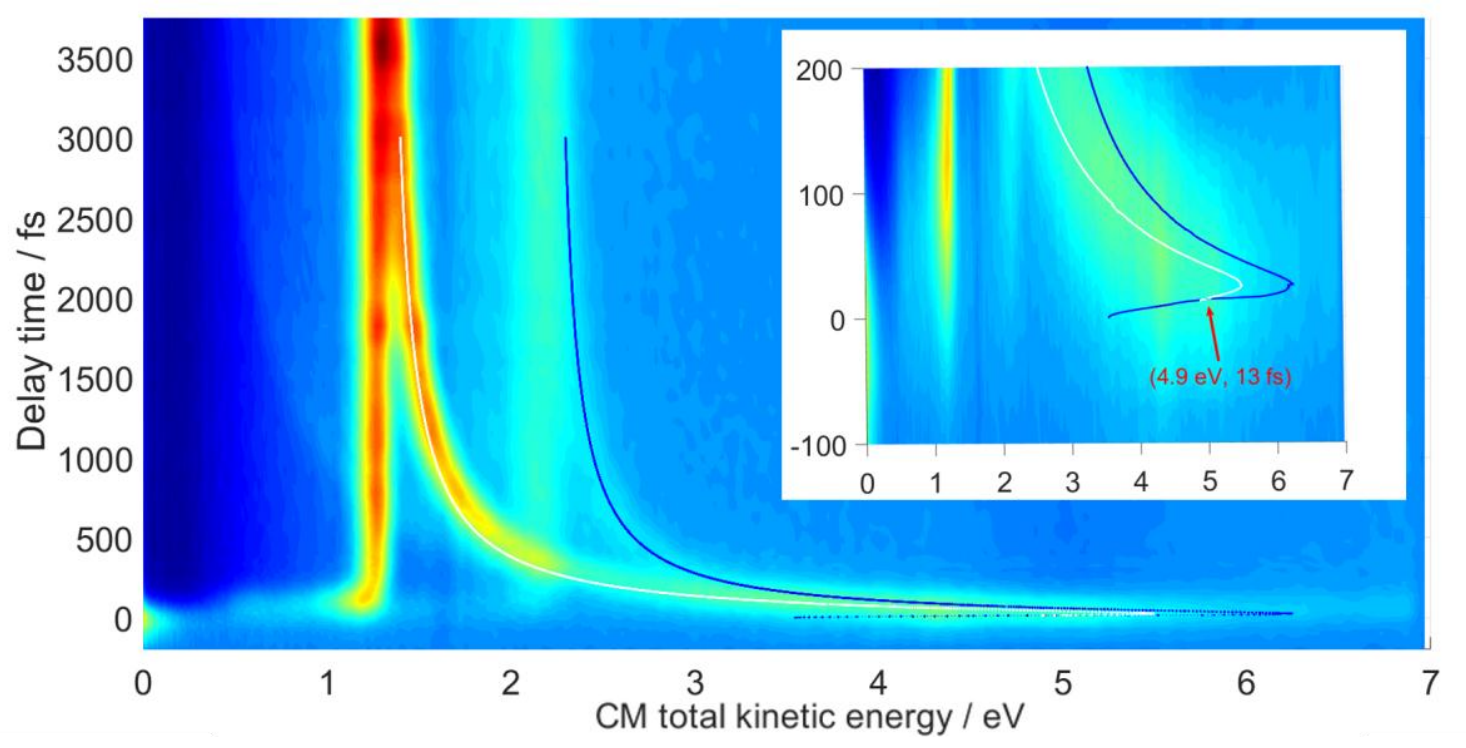

Figure 5. 2D false-color map containing the set of center-of-mass (CM) total KER, as a function of time delay for the $\mathrm{CH}_{3}{ }^{+}$as in Figure 3a. A zoom of the short delay region is depicted in the inset. Solid lines correspond to the trajectory obtained as an average of the 50 trajectories shown in Figure 4. The location of the conical intersection where the bifurcation occurs, is represented by the coordinates $(4.9 \mathrm{eV}, 13 \mathrm{fs})$.

In summary, the results of this work show that Coulomb explosion imaging can be applied as a powerful tool for the visualization of wave packet branching in molecular nonadiabatic dynamical processes, with the advantage of its quasi-direct energy-real space mapping. Beyond this application, the measurement of the Coulomb explosion channels with sufficient energy resolution provides insight into the complex ionization processes leading to the simultaneous excitation of a manifold of cationic states. 


\section{EXPERIMENTAL AND COMPUTATIONAL METHODS}

The experimental setup shares most features with that described in Ref. ${ }^{40}$. A short pulse 268-nm-pump-NIR-probe scheme was employed to irradiate a molecular beam of $\mathrm{CH}_{3} \mathrm{I}$ seeded in He. Velocity map images of the charged fragments were obtained and Abel inverted using the pBasex procedure ${ }^{41}$. High level ab initio calculations in combination with surface hopping semiclassical dynamics ${ }^{42-47}$ were performed to describe the effect of Coulomb explosion during the photodissociation dynamics. Details about both the experimental and computational procedures can be found in the Supporting Information.

\section{ASSOCIATED CONTENT}

Supporting information is available.

\section{ACKNOWLEDGEMENT}

This work has been supported by the Spanish Ministry of Science, Innovation and Universities through grants CTQ2015-65033-P and CTQ2016-75880-P. This research has been carried out within the Unidad Asociada Química Física Molecular between Departamento de Química Física of Universidad Complutense de Madrid and CSIC. The 
facilities provided by the CLUR (Centro de Láseres Ultrarrápidos) are gratefully acknowledged.

\section{REFERENCES}

1. Yarkony, D. R., Diabolical Conical Intersections. Rev. Mod. Phys. 1996, 68 (4), 985-1013.

2. Yarkony, D. R., Conical Intersections: Diabolical and Often Misunderstood. Accounts Chem. Res. 1998, 31 (8), 511-518.

3. Domcke, W.; Yarkony, D. R., Role of Conical Intersections in Molecular Spectroscopy and Photoinduced Chemical Dynamics. In Annual Review of Physical Chemistry, Vol 63, Johnson, M. A.; Martinez, T. J., Eds. Annual Reviews: Palo Alto, 2012; Vol. 63, pp 325-352.

4. Yarkony, D. R., Nonadiabatic Quantum Chemistry-Past, Present, and Future. Chem. Rev. 2012, 112 (1), 481-498.

5. Jasper, A. W.; Zhu, C. Y.; Nangia, S.; Truhlar, D. G., Introductory Lecture: Nonadiabatic Effects in Chemical Dynamics. Faraday Discuss. 2004, 127, 1-22.

6. Stock, G., Classical Description of Nonadiabatic Photoisomerization Processes and Their Real-Time Detection Via Femtosecond Spectroscopy. J. Chem. Phys. 1995, 103 (23), 10015-10029.

7. Blanchet, V.; Zgierski, M. Z.; Seideman, T.; Stolow, A., Discerning Vibronic Molecular Dynamics Using Time-Resolved Photoelectron Spectroscopy. Nature 1999, 401 (6748), 52-54.

8. Farrow, D. A.; Qian, W.; Smith, E. R.; Ferro, A. A.; Jonas, D. M., Polarized Pump-Probe Measurements of Electronic Motion Via a Conical Intersection. J. Chem. Phys. 2008, 128 (14), 22.

9. Larsen, J. J.; Morkbak, N. J.; Olesen, J.; Bjerre, N.; Machholm, M.; Keiding, S. R.; Stapelfeldt, H., Femtosecond Photodissociation Dynamics of I-2 Studied by Ion Imaging. J. Chem. Phys. 1998, 109 (20), 8857-8863.

10. Stapelfeldt, H.; Constant, E.; Sakai, H.; Corkum, P. B., Time-Resolved Coulomb Explosion Imaging: A Method to Measure Structure and Dynamics of Molecular Nuclear Wave Packets. Phys. Rev. A 1998, 58 (1), 426-433.

11. Ergler, T.; Rudenko, A.; Feuerstein, B.; Zrost, K.; Schroter, C. D.; Moshammer, R.; Ullrich, J., Ultrafast Mapping of H-2(+) (D-2(+)) Nuclear Wave Packets Using Time-Resolved Coulomb Explosion Imaging. J. Phys. B-At. Mol. Opt. Phys. 2006, 39 (13), S493-S501.

12. Feuerstein, B.; Ergler, T.; Rudenko, A.; Zrost, K.; Schroter, C. D.; Moshammer, R.; Ullrich, J.; Niederhausen, T.; Thumm, U., Complete Characterization of Molecular Dynamics in Ultrashort Laser Fields. Phys. Rev. Lett. 2007, 99 (15), 4.

13. Kornilov, O.; Eckstein, M.; Rosenblatt, M.; Schulz, C. P.; Motomura, K.; Rouzee, A.; Klei, J.; Foucar, L.; Siano, M.; Lubcke, A.; Schapper, F.; Johnsson, P.; Holland, D. M. P.; Schlatholter, T.; Marchenko, T.; Dusterer, S.; Ueda, K.; Vrakking, M. J. J.; Frasinski, L. J., Coulomb Explosion of Diatomic Molecules in Intense Xuv Fields Mapped by Partial Covariance. J. Phys. B-At. Mol. Opt. Phys. 2013, 46 (16), 11.

14. Gagnon, J.; Lee, K. F.; Rayner, D. M.; Corkum, P. B.; Bhardwaj, V. R., Coincidence Imaging of Polyatomic Molecules Via Laser-Induced Coulomb Explosion. J. Phys. B-At. Mol. Opt. Phys. 2008, 41 (21), 6.

15. Voigtsberger, J.; Zeller, S.; Becht, J.; Neumann, N.; Sturm, F.; Kim, H. K.; Waitz, M.; Trinter, F.; Kunitski, M.; Kalinin, A.; Wu, J.; Schoellkopf, W.; Bressanini, D.; Czasch, A.; Williams, J. B.; 
Ullmann-Pfleger, K.; Schmidt, L. P. H.; Schofffler, M. S.; Grisenti, R. E.; Jahnke, T.; Dorner, R., Imaging the Structure of the Trimer Systems He-4(3) and (Hehe2)-He-3-He-4. Nat. Commun. 2014, 5,6 .

16. Wu, J.; Kunitski, M.; Schmidt, L. P. H.; Jahnke, T.; Dorner, R., Structures of N2ar, O2ar, and O2xe Dimers Studied by Coulomb Explosion Imaging. J. Chem. Phys. 2012, 137 (10), 4.

17. Amini, K.; Boll, R.; Lauer, A.; Burt, M.; Lee, J. W. L.; Christensen, L.; Brausse, F.; Mullins, T.; Savelyev, E.; Ablikim, U.; Berrah, N.; Bomme, C.; Dusterer, S.; Erk, B.; Hoppner, H.; Johnsson, P.; Kierspel, T.; Krecinic, F.; Kupper, J.; Muller, M.; Muller, E.; Redlin, H.; Rouzee, A.; Schirmel, N.; Thogersen, J.; Techert, S.; Toleikis, S.; Treusch, R.; Trippel, S.; Ulmer, A.; Wiese, J.; Vallance, C.; Rudenko, A.; Stapelfeldt, H.; Brouard, M.; Rolles, D., Alignment, Orientation, and Coulomb Explosion of Difluoroiodobenzene Studied with the Pixel Imaging Mass Spectrometry (Pimms) Camera. J. Chem. Phys. 2017, 147 (1), 8.

18. Lee, K. F.; Villeneuve, D. M.; Corkum, P. B.; Stolow, A.; Underwood, J. G., Field-Free ThreeDimensional Alignment of Polyatomic Molecules. Phys. Rev. Lett. 2006, 97 (17), 4.

19. Burt, M.; Amini, K.; Lee, J. W. L.; Christiansen, L.; Johansen, R. R.; Kobayashi, Y.; Pickering, J. D.; Vallance, C.; Brouard, M.; Stapelfeldt, H., Communication: Gas-Phase Structural Isomer Identification by Coulomb Explosion of Aligned Molecules. J. Chem. Phys. 2018, 148 (9), 5.

20. Pitzer, M., How to Determine the Handedness of Single Molecules Using Coulomb Explosion Imaging. J. Phys. B-At. Mol. Opt. Phys. 2017, 50 (15), 17.

21. Stapelfeldt, H.; Constant, E.; Corkum, P. B., Wave-Packet Structure and Dynamics Measured by Coulomb Explosion. Phys. Rev. Lett. 1995, 74 (19), 3780-3783.

22. Amini, K.; Savelyev, E.; Brausse, F.; Berrah, N.; Bomme, C.; Brouard, M.; Burt, M.; Christensen, L.; Dusterer, S.; Erk, B.; Hoppner, H.; Kierspel, T.; Krecinic, F.; Lauer, A.; Lee, J. W. L.; Muller, M.; Muller, E.; Mullins, T.; Redlin, H.; Schirmel, N.; Thogersen, J.; Techert, S.; Toleikis, S.; Treusch, R.; Trippel, S.; Ulmer, A.; Vallance, C.; Wiese, J.; Johnsson, P.; Kupper, J.; Rudenko, A.; Rouzee, A.; Stapelfeldt, H.; Rolles, D.; Boll, R., Photodissociation of Aligned Ch3i and C6h3f2i Molecules Probed with Time-Resolved Coulomb Explosion Imaging by Site-Selective Extreme Ultraviolet lonization. Struct. Dyn.-US 2018, 5 (1), 14.

23. Burt, M.; Boll, R.; Lee, J. W. L.; Amini, K.; Kockert, H.; Vallance, C.; Gentleman, A. S.; Mackenzie, S. R.; Bari, S.; Bomme, C.; Dusterer, S.; Erk, B.; Manschwetus, B.; Muller, E.; Rompotis, D.; Savelyev, E.; Schirmel, N.; Techert, S.; Treusch, R.; Kupper, J.; Trippel, S.; Wiese, J.; Stapelfeldt, H.; de Miranda, B. C.; Guillemin, R.; Ismail, I.; Journel, L.; Marchenko, T.; Palaudoux, J.; Penent, F.; Piancastelli, M. N.; Simon, M.; Travnikova, O.; Brausse, F.; Goldsztejn, G.; Rouzee, A.; Geleoc, M.; Geneaux, R.; Ruchon, T.; Underwood, J.; Holland, D. M. P.; Mereshchenko, A. S.; Olshin, P. K.; Johnsson, P.; Maclot, S.; Lahl, J.; Rudenko, A.; Ziaee, F.; Brouard, M.; Rolles, D., Coulomb-Explosion Imaging of Concurrent Ch2bri Photodissociation Dynamics. Phys. Rev. A 2017, 96 (4), 8.

24. Allum, F.; Burt, M.; Amini, K.; Boll, R.; Köckert, H.; Olshin, P. K.; Bari, S.; Bomme, C.; Brauße, F.; Miranda, B. C. d.; Düsterer, S.; Erk, B.; Géléoc, M.; Geneaux, R.; Gentleman, A. S.; Goldsztejn, G.; Guillemin, R.; Holland, D. M. P.; Ismail, I.; Johnsson, P.; Journel, L.; Küpper, J.; Lahl, J.; Lee, J. W. L.; Maclot, S.; Mackenzie, S. R.; Manschwetus, B.; Mereshchenko, A. S.; Mason, R.; Palaudoux, J.; Piancastelli, M. N.; Penent, F.; Rompotis, D.; Rouzée, A.; Ruchon, T.; Rudenko, A.; Savelyev, E.; Simon, M.; Schirmel, N.; Stapelfeldt, H.; Techert, S.; Travnikova, O.; Trippel, S.; Underwood, J. G.; Vallance, C.; Wiese, J.; Ziaee, F.; Brouard, M.; Marchenko, T.; Rolles, D., Coulomb Explosion Imaging of Ch3i and Ch2cli Photodissociation Dynamics. The Journal of Chemical Physics 2018, 149 (20), 204313.

25. Chandler, D. W.; Houston, P. L., Two-Dimensional Imaging of State-Selected Photodissociation Products Detected by Multiphoton Ionization. J. Chem. Phys. 1987, 87 (2), 14451447. 
26. Eppink, A.; Parker, D. H., Methyl lodide a-Band Decomposition Study by Photofragment Velocity Imaging. J. Chem. Phys. 1998, 109 (12), 4758-4767.

27. Eppink, A.; Parker, D. H., Energy Partitioning Following Photodissociation of Methyl lodide in the a Band: A Velocity Mapping Study. J. Chem. Phys. 1999, 110 (2), 832-844.

28. de Nalda, R.; Izquierdo, J. G.; Dura, J.; Banares, L., Femtosecond Multichannel Photodissociation Dynamics of Ch3i from the a Band by Velocity Map Imaging. J. Chem. Phys. 2007, $126(2), 4$.

29. de Nalda, R.; Dura, J.; Garcia-Vela, A.; Izquierdo, J. G.; Gonzalez-Vazquez, J.; Banares, L., A Detailed Experimental and Theoretical Study of the Femtosecond a-Band Photodissociation of Ch3i. J. Chem. Phys. 2008, 128 (24), 20.

30. Garcia-Vela, A.; de Nalda, R.; Dura, J.; Gonzalez-Vazquez, J.; Banares, L., A 4d Wave Packet Study of the Ch3i Photodissociation in the a-Band. Comparison with Femtosecond Velocity Map Imaging Experiments. J. Chem. Phys. 2011, 135 (15), 12.

31. Amatatsu, Y.; Morokuma, K.; Yabushita, S., Abinitio Potential-Energy Surfaces and Trajectory Studies of a-Band Photodissociation Dynamics - Ch3i Star- Ch3+l and Ch3+l Star. J. Chem. Phys. 1991, 94 (7), 4858-4876.

32. Amatatsu, Y.; Yabushita, S.; Morokuma, K., Full Nine-Dimensional Ab Initio Potential Energy Surfaces and Trajectory Studies of a-Band Photodissociation Dynamics: Ch3i*->Ch3+l, Ch3+l*, and Cd3i*->Cd3+l, Cd3+l*. J. Chem. Phys. 1996, 104 (24), 9783-9794.

33. Xie, D. Q.; Guo, H.; Amatatsu, Y.; Kosloff, R., Three-Dimensional Photodissociation Dynamics of Rotational State Selected Methyl lodide. J. Phys. Chem. A 2000, 104 (5), 1009-1019.

34. Guo, H., 3-Dimensional Photodissociation Dynamics of Methyl-lodide. J. Chem. Phys. 1992, $96(9), 6629-6642$.

35. Evenhuis, C. R.; Manthe, U., Photodissociation of Ch3i: A Full-Dimensional (9d) Quantum Dynamics Study. J. Phys. Chem. A 2011, 115 (23), 5992-6001.

36. Corrales, M. E.; Gitzinger, G.; Gonzalez-Vazquez, J.; Loriot, V.; de Nalda, R.; Banares, L., Velocity Map Imaging and Theoretical Study of the Coulomb Explosion of Ch3i under Intense Femtosecond Ir Pulses. J. Phys. Chem. A 2012, 116 (11), 2669-2677.

37. Wang, Y. M.; Zhang, S.; Wei, Z. R.; Zhang, B., Velocity Map Imaging of Dissociative Ionization and Coulomb Explosion of Ch3i Induced by a Femtosecond Laser. J. Phys. Chem. A 2008, 112 (17), 3846-3851.

38. Dura, J.; de Nalda, R.; Alvarez, J.; Izquierdo, J. G.; Amaral, G. A.; Banares, L., Femtosecond Transition-State Imaging of the a-Band Ch3i Photodissociation. ChemPhysChem 2008, 9 (9), 12451249.

39. Dura, J.; de Nalda, R.; Amaral, G. A.; Banares, L., Imaging Transient Species in the Femtosecond a-Band Photodissociation of Ch3i. J. Chem. Phys. 2009, 131 (13), 14.

40. Corrales, M. E.; Gonzalez-Vazquez, J.; Balerdi, G.; Sola, I. R.; de Nalda, R.; Banares, L., Control of Ultrafast Molecular Photodissociation by Laser-Field-Induced Potentials. Nat. Chem. 2014, 6 (9), 785-790.

41. Garcia, G. A.; Nahon, L.; Powis, I., Two-Dimensional Charged Particle Image Inversion Using a Polar Basis Function Expansion. Rev. Sci. Instrum. 2004, 75 (11), 4989-4996.

42. Poullain, S. M.; Chicharro, D. V.; Navarro, E.; Rubio-Lago, L.; Gonzalez-Vazquez, J.; Banares, L., Photodissociation Dynamics of Bromoiodomethane from the First and Second Absorption Bands. A Combined Velocity Map and Slice Imaging Study. Phys. Chem. Chem. Phys. 2018, 20 (5), 3490-3503.

43. Murillo-Sanchez, M. L.; Poullain, S. M.; Gonzalez-Vazquez, J.; Corrales, M. E.; Balerdi, G.; Banares, L., Femtosecond Photodissociation Dynamics of Chloroiodomethane in the First Absorption Band. Chem. Phys. Lett. 2017, 683, 22-28. 
44. Chicharro, D. V.; Poullain, S. M.; Gonzalez-Vazquez, J.; Banares, L., Slice Imaging of the Uv Photodissociation of $\mathrm{Ch} 2 \mathrm{brcl}$ from the Maximum of the First Absorption Band. J. Chem. Phys. 2017, $147(1), 10$.

45. Murillo-Sanchez, M. L.; Poullain, S. M.; Bajo, J. J.; Corrales, M. E.; Gonzalez-Vazquez, J.; Sola, I. R.; Banares, L., Halogen-Atom Effect on the Ultrafast Photodissociation Dynamics of the Dihalomethanes Ch2icl and Ch2bri. Phys. Chem. Chem. Phys. 2018, 20 (32), 20766-20778.

46. Richter, M.; Marquetand, P.; Gonzalez-Vazquez, J.; Sola, I.; Gonzalez, L., Sharc: Ab Initio Molecular Dynamics with Surface Hopping in the Adiabatic Representation Including Arbitrary Couplings. J. Chem. Theory Comput. 2011, 7 (5), 1253-1258.

47. Marquetand, P.; Richter, M.; Gonzalez-Vazquez, J.; Sola, I.; Gonzalez, L., Nonadiabatic Ab Initio Molecular Dynamics Including Spin-Orbit Coupling and Laser Fields. Faraday Discuss. 2011, 153, 261-273. 